\title{
What is 'anti' about anti-reaches? Reference frames selectively affect reaction times and endpoint variability
}

\author{
Stephanie Westendorff · Alexander Gail
}

Received: 15 June 2010 / Accepted: 25 October 2010 / Published online: 13 November 2010

(c) The Author(s) 2010. This article is published with open access at Springerlink.com

\begin{abstract}
Reach movement planning involves the representation of spatial target information in different reference frames. Neurons at parietal and premotor stages of the cortical sensorimotor system represent target information in eyeor hand-centered reference frames, respectively. How the different neuronal representations affect behavioral parameters of motor planning and control, i.e. which stage of neural representation is relevant for which aspect of behavior, is not obvious from the physiology. Here, we test with a behavioral experiment if different kinematic movement parameters are affected to a different degree by either an eye- or hand-reference frame. We used a generalized anti-reach task to test the influence of stimulus-response compatibility (SRC) in eyeand hand-reference frames on reach reaction times, movement times, and endpoint variability. While in a standard anti-reach task, the SRC is identical in the eye- and hand-reference frames, we could separate SRC for the two reference frames. We found that reaction times were influenced by the SRC in eye- and hand-reference frame. In contrast, movement times were only influenced by the SRC in hand-reference frame, and endpoint variability was only influenced by the SRC in eye-reference frame. Since movement time and endpoint variability are the result of planning and control processes, while reaction times are consequences of only the planning process, we suggest that SRC effects on reaction times are highly suited to investigate reference frames of movement planning, and that eye- and hand-reference frames have distinct effects on different phases of motor action and different kinematic movement parameters.
\end{abstract}

\footnotetext{
S. Westendorff · A. Gail $(\bowtie)$

Bernstein Center for Computational Neuroscience Göttingen, German Primate Center, Kellnerweg 4,

37077 Göttingen, Germany

e-mail: agail@gwdg.de
}

Keywords Reach planning $\cdot$ Stimulus-response compatibility $\cdot$ Reference frames $\cdot$ Sensorimotor transformation $\cdot$ Eye-hand coordination

\section{Introduction}

To successfully plan and control goal-directed reach movements, one has to estimate the motor error between the current hand position and the target position. One assumption is that in order to compute the difference vector, the hand and target positions have to be represented in a common reference frame (Buneo and Andersen 2006). This might be a representation in eye-, shoulder- or some other body-centered reference frame. A lesion study (Khan et al. 2005) and imaging data (Medendorp et al. 2003) from humans and electrophysiological studies in monkeys (Batista et al. 1999; Buneo et al. 2002; Pesaran et al. 2006) showed that the parietal reach region (PRR) of the posterior parietal cortex encodes planned reach target locations predominantly relative to the direction of the gaze. This suggests that PRR represents a stage of reach planning prior to the definition of the motor error in a hand-reference frame. In parietal area 5 (Buneo et al. 2002) and the dorsal premotor cortex (Batista et al. 2007; Pesaran et al. 2006), the reach target location in a hand-reference frame contributes stronger to the spatial representations, often resulting in a combined encoding of eye, hand and target position.

Which level of processing finally is responsible for which aspect of behavioral performance is not clear from these neurophysiological observations. There is psychophysical support for the encoding of remembered reach target locations in an eye-reference frame (Beurze et al. 2006; Henriques et al. 1998; Sorrento and Henriques 2008), while other experiments showed an influence of 
hand-reference frame (Bock and Eckmiller 1986), or indicate that reach planning can be achieved in a combination of multiple reference frames (McGuire and Sabes 2009). The latter result suggests that the contribution of each reference frame depends on the available information in that reference frame. If the reach target was defined visually, the eye-centered representation was weighted stronger than a body-centered representation, and vice versa, if the reach target was defined by a proprioceptive target, the bodycentered representation gained more weight. These previous psychophysical studies used systematic reach endpoint errors for determining the reference frame of movement planning. Endpoint errors not only depend on movement planning, but also motor control. Similarly, movement times (MTs) and endpoint variability (EVs) reflect both planning and control processes of the movement. Reaction times (RTs), in contrast, cannot be influenced by motor control processes, since they are measured before onset of the movement and thereby allow isolating the influence of reference frames during the planning process.

We tested the hypothesis that different movement parameters are differently influenced by an eye- and handcentered reference frames. Alternatively, a single reference frames could affect multiple parameters of movement planning and control in the same way, e.g. resulting from a task-specific cognitive strategy or selective availability of different sensory input signals (McGuire and Sabes 2009). Going beyond previous studies, we designed an experiment in which we could compare the influence of eye- and handcentered reference frames on RTs, MTs and EVs within the same behavioral task.

We designed a new pro-/anti-reach task with which we could modify spatial stimulus-response compatibility (SRC) separately in an eye- and hand-reference frame (Lamberts et al. 1992; Nicoletti and Umilta 1989; Umilta and Liotti 1987). The idea is that SRC in a certain reference frame (e.g. eye-reference frame) only should have an effect on a certain parameter (e.g. RT) if this reference frame is functionally relevant for the respective movement parameter. Furthermore, the idea is that the reference frame which contributes to SRC effects in a certain movement parameter is also the reference frame of the neuronal representations underlying this movement parameter. In this sense, an influence of a certain reference frame on a behavioral parameter could help to relate behavioral parameters to brain areas with activity pattern in the same reference frame.

Spatial compatibility between the instruction stimulus (cue) and the associated behavioral response is known to influence RTs in various types of tasks. Subjects are in general faster if the spatial information contained in a visual cue matches spatial response parameters, independent of the exact type of movement to be performed (Duncan 1977; Fitts and Deininger 1954; Fitts and Seeger 1953; Georgop- oulos et al. 1989; Hommel 1996; Lamberts et al. 1992; Morin and Grant 1955; Nicoletti and Umilta 1984; Proctor and $\mathrm{Vu}$ 2002; Shaffer 1965). In pro-/anti-paradigms (Crammond and Kalaska 1994; Everling et al. 1998; Fischer and Weber 1992; Gail and Andersen 2006; Hallett 1978; Zhang and Barash 2000), a pro-response is directed toward a spatial stimulus, whereas an anti-response is directed opposite to the spatial stimulus. In contrast to button-presses or joystick experiments, subjects in pro-/anti-reach tasks execute reach movements in the same workspace as the visual instructions are given, which makes eye- and hand-visuospatial reference frames more comparable.

In a standard pro-/anti-reach task, the SRC is identical in the eye- and hand-reference frame. We developed a generalized pro-/anti-reach task to dissociate the influence of eye- and hand-reference frame on SRC effects. With the task design, we could define reaches that were compatible in one reference frame, but not the other, and vice versa. Viewed from a slightly different perspective, the generalized pro-/anti-reach task allows answering the question of what makes an anti-reach incompatible, the incompatibility of cue and response in the eye- or hand-reference frame. We found that SRC in eye- and hand-reference frames affected RTs, MTs and EVs in a distinct manner, indicating that different aspects of movement planning and control are influenced by the two reference frames to a different degree.

\section{Methods}

Subjects

Sixteen right-handed subjects (7 females, 22-38 years) with normal or corrected-to-normal vision participated in the main experiment, 15 (8 females, 21-27 years) in a control experiment. All were naïve with respect to the objective of the study. Detailed written instructions were given to the subjects before the experiment. Subjects had the opportunity to get familiar with the setup and practice the task for about $15 \mathrm{~min}$. All subjects had a success rate higher than $70 \%$ during training, which was a prerequisite for participation in the recording session. Experiments were in accordance with institutional guidelines for experiments with humans and adhered to the principles of the Declaration of Helsinki. All subjects gave their informed consent prior to their inclusion in the study.

\section{Generalized pro- and anti-reach task}

In a choice reaction-time task subjects had to perform reaches with their preferred hand on a touch screen. Reaches were instructed by two visual cues: A colored context cue (green or blue square frame around eye-fixation 
point, $\sim 3^{\circ}$ visual angle (VA) edge length) indicated whether the subjects had to make a reach into the same (pro-reach, green) or opposite (anti-reach, blue) direction of a spatial cue. The spatial cue (white circular patch, diameter of $\sim 3^{\circ} \mathrm{VA}$ ) instructed the movement direction in an eyecentered reference frame.

Eye- and hand-fixation stimuli were presented at $\pm 5 \mathrm{~cm}$ $\left(7^{\circ} \mathrm{VA}\right)$ relative to the screen center, spatial cues at $0 \mathrm{~cm}$ (screen center) or $\pm 10 \mathrm{~cm}$. Over the whole experiment, the three potential spatial cue positions were also potential reach target positions. But in each individual trial only the two cue positions at $\pm 5 \mathrm{~cm}$ to the eye stimulus served as potential cue positions. Therefore, the spatial cue appeared always at the same visual eccentricity. Similarly, in each trial only the two target positions at $5 \mathrm{~cm}$ to the left or right of the hand-fixation position served as potential reach goals, such that all reaches had the same reach amplitude of $5 \mathrm{~cm}$, and a $50 \%$ probability of leftward or rightward direction.

Half of the trials were standard pro-/anti-trials. In standard pro-/anti-trials, the eye- and hand-fixation points were identical, either at the $+5 \mathrm{~cm}$ (right fixation) or at the $-5 \mathrm{~cm}$ (left fixation) screen position. A spatial cue appeared either left or right of the fixation points, and subjects had to make a movement in the same (pro) or opposite (anti) direction. A standard pro-reach is compatible in eye- and hand-reference frame, since cue and target are identical. A standard anti-reach is incompatible in eye- and hand-reference frame, since cue and target lie in opposite directions with respect to gaze and hand starting position.

The other half of the trials were generalized pro-/antitrials in which the compatibility in an eye-reference frame can be dissociated from compatibility in a hand-reference frame. In the generalized pro-/anti-reach, eye- and handfixation points were separated. Subjects had to eye-fixate at the $-5 \mathrm{~cm}$ and hand-fixate at the $+5 \mathrm{~cm}$ screen position, or vice versa. The instruction for the generalized pro-/antireach was the same as for the standard pro-/anti-reach: Subjects had to reach in the same or opposite direction of the cue. Note, for the purpose of instructing the subjects, the spatial cue direction was defined relative to the eyefixation position (solid black arrow; Fig. 1a), and the reach direction was defined relative the to hand-fixation position (open gray arrow). For the purpose of analyzing the data, and different from the task instruction, a trial was defined as compatible/incompatible in eye-reference frame if the direction of the spatial cue and the reach goal both were the same/opposite in relation to the eye-fixation stimulus (black arrows, Fig. 1b). And a trial was defined as compatible/ incompatible in hand-reference frame if the direction of the a
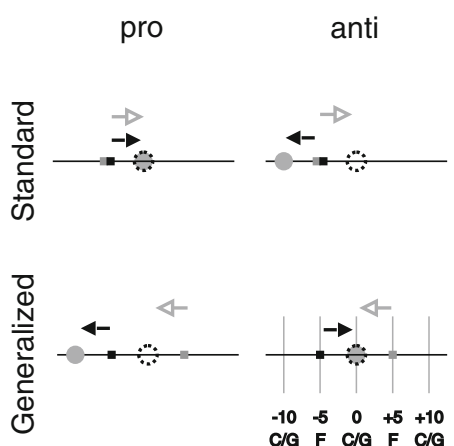

b

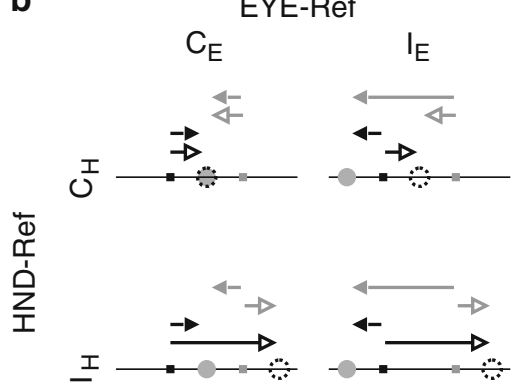

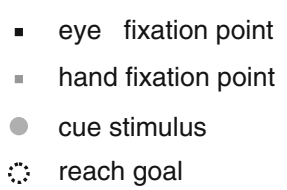

- eye fixation point

- hand fixation point

reach goa $\rightarrow$ direction of spatial cue in eye reference frame

$\rightarrow$ direction of reach goal in eye reference frame

$\rightarrow$ direction of spatial cue in hand reference frame

$\rightarrow$ direction of reach goal in hand reference frame
Fig. 1 Spatial layout of the generalized pro-/anti-reach task. a General task design in standard and generalized trials. Squares depict positions where eye- and/or hand-fixation points $(F)$ were presented. Gray circles depict the positions where the spatial cue $(C)$ was presented. Dotted circles depict the positions of the reach goal $(G)$. In the lower right panel, the $5 \mathrm{~cm}$ raster is illustrated at which the fixation points, cues, and goals could be positioned. For simplicity, each panel only shows one out of four possible spatial configurations (fixation left/ right, cue left/right) for each of the four task conditions (pro/ anti $\times$ standard/generalized). The task design consists of a total of 16 conditions. In a, black arrows indicate the direction of the spatial cue relative to eye fixation, and the gray arrows the direction of the reach goal relative to hand fixation. In pro-trials (per definition) both arrows point in the same direction, whereas in anti-trials, they point in opposite directions. b Four example trials in the generalized task condition, which illustrate the $2 \times 2$ variations of the SR compatibility in the eye-reference frame (black arrows) and in the hand-reference frame (gray arrows). The open arrows show the direction of the spatial cue, the solid arrows show the direction of the reach goal 
spatial cue and the reach goal were the same/opposite in relation to the hand-fixation stimulus (gray arrows). The separation of eye fixation from hand fixation in the generalized conditions, in combination with the pro-/anti-rule, leads to reaches, which were compatible in eye-reference frame, but incompatible in hand-reference frame $\left(\mathrm{C}_{\mathrm{E}} \mathrm{I}_{\mathrm{H}}\right.$; Fig. 1b), and reaches, which were compatible in hand-reference frame, but incompatible in eye-reference frame $\left(\mathrm{I}_{\mathrm{E}} \mathrm{C}_{\mathrm{H}}\right)$. There were also reaches, which were compatible in eyeand hand-reference frame $\left(\mathrm{C}_{\mathrm{E}} \mathrm{C}_{\mathrm{H}}\right)$ and reaches, which were incompatible in eye- and hand-reference frame $\left(\mathrm{I}_{\mathrm{E}} \mathrm{I}_{\mathrm{H}}\right)$, like in the standard pro-/anti-task. Note that the compatibility of cue and reach goal in either reference frame refers only to compatibility with respect to the direction of cue and reach goal. The distance of the cue from the eye-/hand-fixation point can be different than the distance of the reach goal from the respective fixation point (see arrow length in Fig. 1b).

The timeline of the reaction-time task is shown in Fig. 2. The subject initiated a trial by fixating a small, red fixation spot and touching a white hand target (fixation period). After a random delay $(0.5-1.0 \mathrm{~s})$, the context cue was briefly flashed (pre-cue period, $0.2 \mathrm{~s}$ ). The context cue was presented early to induce the effects of compatibility even in a paradigm in which compatible and incompatible conditions were interleaved randomly (de Jong 1995; Proctor and

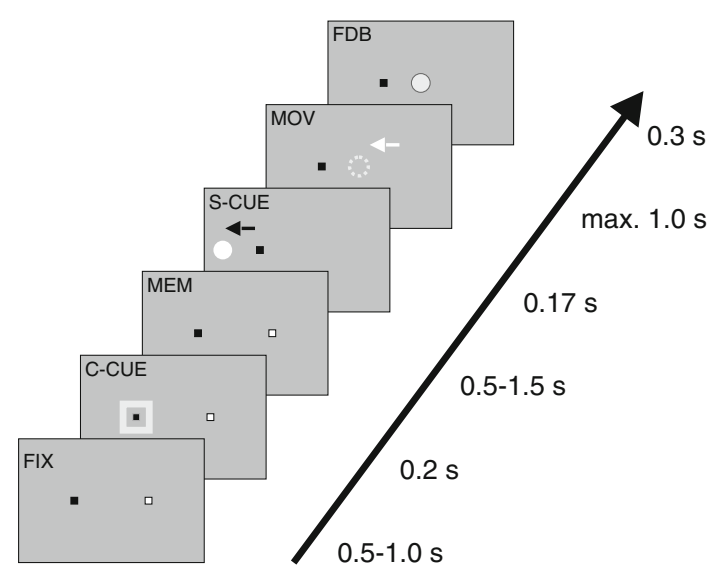

Fig. 2 Timeline of the generalized pro-/anti-reach task. The subject had to direct gaze to a small red square throughout the trial. A flashed context cue (C-CUE, frame around the eye-fixation) instructed whether to prepare a pro- or anti- reach. In the experiment, a green frame instructed a pro-reach and a blue frame instructed an anti-reach. The reach goal (dotted circle, not visible to the subject) was defined by the combination of the context cue and a spatial cue (S-CUE, white circle), which was flashed left or right of the eye fixation after a variable memory period $(M E M)$. Visual feedback $(F D B)$ appeared only after the subject touched the correct reach goal on the screen. Pro- and anti-reaches were defined as a reaches relative to hand fixation (symbolized by white arrow, not shown to subjects) in the same (pro) or opposite (anti) direction as the spatial cue was relative to eye fixation (symbolized by black arrow, not shown to subjects). The example shows a generalized pro-trial
Vu 2002; Shaffer 1965). For a variable duration, the subject had to keep the hand fixation (memory period, $0.5-1.5 \mathrm{~s}$ ) until the hand target turned off (go-signal) and simultaneously the spatial cue was flashed (go-cue period, $0.17 \mathrm{~s}$ ). After the go-signal, the subject had to reach toward the cued reach goal location (movement period, max. $1.0 \mathrm{~s}$, including reaction and movement time). The hand had to be kept at the reach goal location (feedback period, $0.3 \mathrm{~s}$ ) to successfully finish the trial. The subject received visual feedback about the correct reach goal, consisting of a circular patch stimulus at the reach goal location presented immediately after acquiring the desired position. If the subject did not reach the goal location before the maximum movement period expired, then the trial was aborted immediately. An auditory feedback (high/low pitch tone) indicated whether the trial was correct or not.

All parameters of the task (standard/generalized, pro/anti, cue left/cue right, fixation left/ fixation right) were randomly interleaved. Only correct trials were analysed, and each subject performed about 20 correct trials per condition.

\section{Visual display and behavioral control}

Visual instruction stimuli were presented on a LCD screen (19" ViewSonic VX922) mounted behind a touch screen (IntelliTouch, ELO Systems, CA, USA). Custom-written display software $(\mathrm{C}++)$ was controlled via a real-time LabView control program running on a PXI computer (National Instruments). The display of the stimuli was synchronized with the vertical synchronization of the screen to avoid latency jitter. Visual display latencies were recorded with a photodiode and corrected for in the data analysis. All visual instruction stimuli had high contrast and were readily visible. Subjects were seated in front of a fronto-parallel touch screen $(40 \mathrm{~cm}$ distance from eye, screen center at eye level) with a chinrest to minimize head movements. Reaches were not constrained in any specific manner other than the touch positions on the touch screen.

Hand position was registered with the touch screen and monitored within the real-time control software. The hand fixation and reach targets had to be continuously touched within a tolerance window of typically $3 \mathrm{~cm}\left(4.0^{\circ} \mathrm{VA}\right)$ radius. Otherwise the trial was immediately aborted. Reaction time (RT) was defined as the time between the go-signal and the subject's release of the touch screen from the fixation position. Movement time (MT) was defined as the time between the release and re-acquisition of the touch screen at a target position. Endpoint variability (EV) was defined as variable reach error, i.e. the distance of reach endpoint in each trial to the mean reach endpoint to the same reach target. EVs within each subject were calculated separately for the $x$ - and $y$-dimension. RTs, MTs and EVs were calculated separately for each task condition. 
To control for possible effects of eye movements in the main dataset, a second group of 15 subjects was recorded in the same task but with eye movements being constrained. With this control experiment, we wanted to exclude the possibility that compatibility effects could be explained by reflexive saccades of the subjects toward the spatial cue location before executing the reach toward the reach goal. Such behavior would cause a delay in reach responses, since eye movements often lead and predict hand movements (Ariff et al. 2002), and reaction times in incompatible trials would be artificially prolonged, if the subjects executed two saccades (one reflexive to the spatial cue position and one corrective to the reach goal) before the start of the reach. In the control experiment, gaze direction was constrained to a tolerance window of $2 \mathrm{~cm}\left(\sim 2.8^{\circ} \mathrm{VA}\right)$ radius, otherwise the trial was immediately aborted $(500 \mathrm{~Hz}$ IR camera, SMI, Teltow, Germany).

\section{Data analysis}

In the standard pro-/anti-task, compatibility and incompatibility in eye- and hand-reference frames covary. We tested for the compatibility effects in eye- and hand-reference frames by comparing RTs, MTs and EVs in all compatible against all incompatible trials, independent of the direction of cue and reach (left/right) and laterality of fixation (left/ right), using a $t$ test. The main research question of our experiment regards the separation of compatibility effects in an eye-centered from compatibility effects in a hand-centered reference frame. For this, we tested RTs, MTs, and EVs with a repeated-measurement two-way ANOVA with the factors eye- and hand-reference frame in the generalized pro-/anti-task. Figure $1 \mathrm{~b}$ illustrates the $2 \times 2$ design of the ANOVA. The columns depict the conditions, which are compatible (left column) and incompatible (right column) in an eye-centered reference frame, whereas the rows depict conditions, which are compatible (upper row) and incompatible (lower row) in a hand-centered reference frame. Direction of cue and reach (left/right) and laterality of eye and hand fixation (left/right) were not treated as factors. Note, since in the standard task the compatibility in eyeand hand-reference frame always covary, we cannot simply expand the ANOVA to a third factor "standard/generalized", but instead have to analyse both data sets separately.

\section{Results}

The average success rate of the subjects in standard trials was $88 \pm 2 \%$ in pro and $88 \pm 1 \%$ in anti-reaches (mean $\pm \mathrm{SEM})$. First, we tested standard pro- and antireaches for SRC effects across all sample subjects (Fig. 3a, dashed line). RTs in standard pro-trials were on average faster than in standard anti-trials (pro: $326 \pm 11 \mathrm{~ms}$, anti: $355 \pm 11 \mathrm{~ms}$, mean $\pm \mathrm{SEM}, N=16, P=0.00016$, paired $t$ test). In the standard conditions, compatibility in eye- and hand-reference frame was always the same. To test compatibility effects in eye- or hand-reference frame, we analysed the generalized pro-/anti-conditions. In generalized trials, cue and reach goal were compatible in either eye- or handreference frame, but not in the other $\left(\mathrm{C}_{\mathrm{E}} \mathrm{I}_{\mathrm{H}}\right.$ or $\left.\mathrm{I}_{\mathrm{E}} \mathrm{C}_{\mathrm{H}}\right)$, or they were compatible in both reference frames $\left(\mathrm{C}_{\mathrm{E}} \mathrm{C}_{\mathrm{H}}\right)$, or they were incompatible in both reference frames $\left(\mathrm{I}_{\mathrm{E}} \mathrm{I}_{\mathrm{H}}\right)$. The average success rate was similar in those conditions $\left(\mathrm{C}_{\mathrm{E}} \mathrm{C}_{\mathrm{H}}\right.$ : $87 \pm 2 \% ; \mathrm{C}_{\mathrm{E}} \mathrm{I}_{\mathrm{H}}: 84 \pm 2 \% ; \mathrm{I}_{\mathrm{E}} \mathrm{C}_{\mathrm{H}}: 89 \pm 2 \% ; \mathrm{I}_{\mathrm{E}} \mathrm{I}_{\mathrm{H}}: 87 \pm 2 \%$ ). Figure 3 shows the RT results across all sample subjects. RTs were fastest if spatial cue and reach goal were compatible in both reference frames $\left(\mathrm{C}_{\mathrm{E}} \mathrm{C}_{\mathrm{H}}: 367 \pm 18 \mathrm{~ms}\right)$, intermediate if cue and goal were compatible in one reference frame but incompatible in the other reference frame $\left(\mathrm{I}_{\mathrm{E}} \mathrm{C}_{\mathrm{H}}\right.$ : $415 \pm 13 \mathrm{~ms} ; \mathrm{C}_{\mathrm{E}} \mathrm{I}_{\mathrm{H}}: 414 \pm 16 \mathrm{~ms}$ ), and slowest if cue and goal were incompatible in both reference frames $\left(\mathrm{I}_{\mathrm{E}} \mathrm{I}_{\mathrm{H}}\right.$ : $447 \pm 16 \mathrm{~ms}$ ). A repeated-measurement two-way ANOVA with factors eye compatibility and hand compatibility revealed a main effect for both, eye $(F(1,15)=22.558$, $\mathrm{MSE}=1,163, P=0.0003)$ and hand $(F(1,15)=19.46$, MSE $=1,263, P=0.0005)$ reference frame, with no interaction $(F=0.57, \mathrm{MSE}=1,545, P=0.46)$ in generalized trials. Standard trials were faster than generalized trials $\left(P<10^{-8}\right.$, paired $t$ test $)$.

Figure $3 \mathrm{~b}$ shows the average differences of all paired group comparisons. We conducted post hoc comparisons between all groups using paired $t$ tests $\left(\alpha_{\text {corr }}=0.0083\right.$ for $n=6$ multiple comparisons). The significances are indicated in Fig. 3b. Additionally, one can take from these post hoc comparisons that (a) the hand-compatibility effect, i.e. the RT difference between trials, which were compatible and trials, which were incompatible in a hand-centered reference frame, in eye-compatible trials $\left(\mathrm{C}_{\mathrm{E}} \mathrm{C}_{\mathrm{H}}\right.$ vs. $\mathrm{C}_{\mathrm{E}} \mathrm{I}_{\mathrm{H}}$; $47 \pm 14 \mathrm{~ms}$ ) is about equal to the eye-compatibility effect, i.e. the RT difference between trials which were compatible and trials which were incompatible in an eye-centered reference frame, in hand-compatible trials $\left(\mathrm{C}_{\mathrm{E}} \mathrm{C}_{\mathrm{H}}\right.$ vs. $\mathrm{I}_{\mathrm{E}} \mathrm{C}_{\mathrm{H}}$; $48 \pm 15 \mathrm{~ms}$ ), and (b) the hand-compatibility effect in eyeincompatible trials $\left(\mathrm{I}_{\mathrm{E}} \mathrm{C}_{\mathrm{H}}\right.$ vs. $\left.\mathrm{I}_{\mathrm{E}} \mathrm{I}_{\mathrm{H}} ; 32 \pm 13 \mathrm{~ms}\right)$ is about equal to the eye-compatibility effect in hand-incompatible trials $\left(\mathrm{C}_{\mathrm{E}} \mathrm{I}_{\mathrm{H}}\right.$ vs. $\mathrm{I}_{\mathrm{E}} \mathrm{I}_{\mathrm{H}} ; 33 \pm 10 \mathrm{~ms}$; Fig. 3b). The first and the second bar of Fig. 3b show that the difference between $\mathrm{I}_{\mathrm{E}} \mathrm{I}_{\mathrm{H}}$ and $\mathrm{C}_{\mathrm{E}} \mathrm{C}_{\mathrm{H}}$ trials is less for standard $(29 \pm 6 \mathrm{~ms})$ then for generalized $(80 \pm 14 \mathrm{~ms})$ trials $(P=0.0023$, paired $t$ test). In summary, this means that RTs increase due to incompatibility in eye-reference frame and due to incompatibility in hand-reference frame and that both effects were about equally large.

Figure 4 shows the influences of different reference frames on SRC effects in MTs. The analysis is equivalent to 


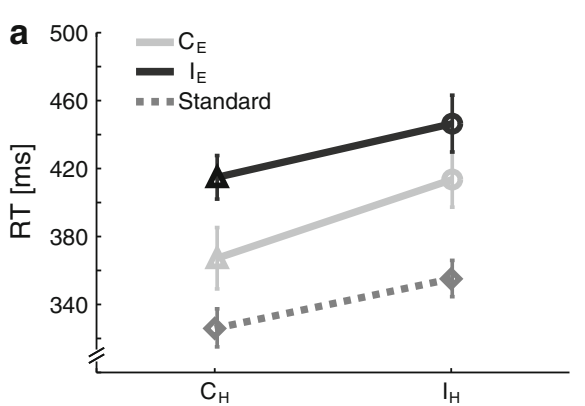

Fig. 3 Influence of SR compatibility in eye- and hand-reference frames on reach reaction times $(R T s)$. a Average (mean \pm SEM) RTs for the different combinations of compatibility/incompatibility in the hand-reference frame $\left(\mathrm{C}_{\mathrm{H}}\right.$ (triangles $) / \mathrm{I}_{\mathrm{H}}$ (circles)) and compatibility/ incompatibility in the eye-reference frame $\left(\mathrm{C}_{\mathrm{E}}\right.$ (light gray) $/ \mathrm{I}_{\mathrm{E}}($ black $\left.)\right)$. RTs in the standard trials are plotted separately (dashed, dark gray

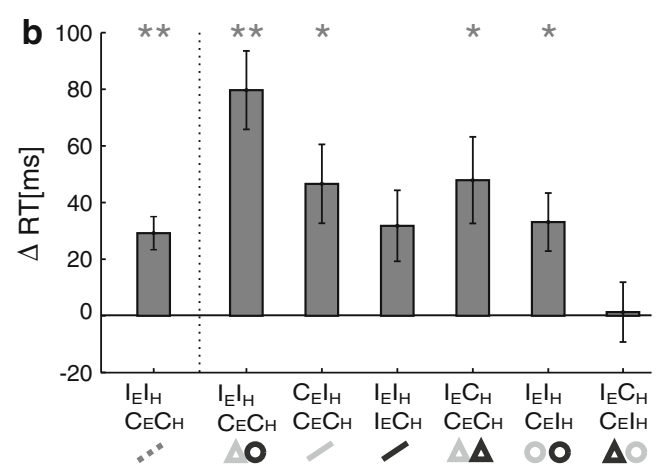

curve). Note that for the standard trials eye and hand compatibility are identical. b Average inter-subject difference between the compatible and incompatible trials in the standard condition (1st bar) and between all possible combination of compatibility conditions in generalized trials. $* P<0.05$; $* * P<0.01$, paired $t$ test, Bonferroni corrected
Fig. 4 Influence of SR compatibility in eye- and hand-reference frames on reach movement times $(M T s)$. Conventions are the same as for Fig. 3

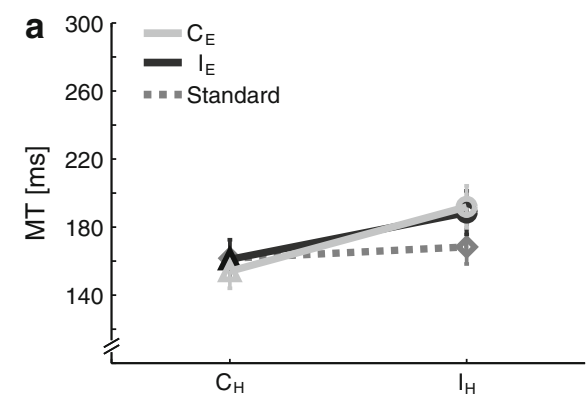

RTs in Fig. 3. MTs in standard pro-reaches $(162 \pm 11 \mathrm{~ms})$ were faster than in standard anti-reaches $(168 \pm 10 \mathrm{~ms}$; $P=0.042$, paired $t$ test; Fig. $4 \mathrm{a}$, dashed line; Fig. $4 \mathrm{~b}$, left bar). In generalized trials, the repeated-measurement twoway ANOVA with factors eye- and hand-reference frame revealed a main effect for hand-reference frame $(F(1,15)=$ 69.00, MSE $\left.=248, P<10^{-4}\right)$, but no effect for eye-reference frame $(F(1,15)=0.25$, MSE $=201, P=0.62)$, and no interaction $(F(1,15)=1.48, \mathrm{MSE}=288, P=0.24)$. Post hoc tests revealed that there was a hand-compatibility effect in both eye-compatible $\left(P<10^{-5}, \alpha_{\text {corr }}=0.0083\right)$ and eye-incompatible trials $(P=0.0003)$, while there was an eye-compatibility effect neither in hand-compatible $(P=0.14$, $)$ nor in handincompatible trials $(P=0.61)$.

The influence of SRC in eye- and hand-reference frame on EVs in the relevant horizontal $x$-dimension is shown in Fig. 5. Standard pro-trials show smaller EVs $(0.47 \pm$ $0.02 \mathrm{~cm}$ than standard anti-trials $(0.58 \pm 0.04 \mathrm{~cm}, P=$ 0.0017, paired $t$ test; Fig. 5a, dashed line; Fig. 5b, left bar). In generalized trials, the repeated-measurement two-way ANOVA showed a significant main effect of eye-reference frame $\left(F(1,15)=48.8, \mathrm{MSE}=0.003, P<10^{-4}\right)$, but no effect of hand-reference frame $(F(1,15)=1.19, \mathrm{MSE}=0.022$,
$P=0.29)$, and no interaction $(F(1,15)=1.34, \quad \mathrm{MSE}=$ $0.009, P=0.27)$. Post hoc tests revealed that there was no hand-compatibility effect in either eye-compatible $(P=0.077$, $\left.\alpha_{\text {corr }}=0.0083\right)$ or eye-incompatible trials $(P=0.84)$. There was an eye-compatibility effect in hand-compatible $(P=0.0005)$ but not hand-incompatible trials $(P=0.027)$. In our task design reach goal position varied only in the $x$-dimension. Accordingly, we did not see any effect of eyeor hand-reference frame on the EV in the $y$-dimension (data not shown).

\section{Constraint of eye movements}

The results described earlier were obtained while subjects were instructed to keep ocular fixation on the fixation spot, but without registering the actual eye movements. If subjects could not reliably follow the instruction of keeping their gaze fixed, but, for example, made many unvoluntary saccades toward the flashed spatial cue, then such saccades could have interfered with the reach initialization, and could thereby have confounded RT data.

We recorded 15 additional subjects (14 new, 1 from the previous sample) in the same task while constraining their 
Fig. 5 Influence of eye- and hand-reference frames on reach endpoint variability $(E V)$. Conventions are the same as in Fig. 3

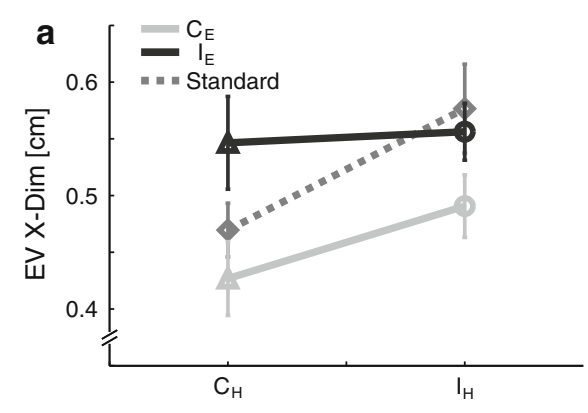

gaze in real time (see "Methods"). The control experiment turned out to be challenging to the subjects. On average across subjects, the success rate was $79 \pm 3 \%$ in standard pro-trials and $80 \pm 3 \%$ in standard anti-trials. The average success rate in the standard trials of the control experiment was lower than in the main experiment $(P=0.0055, t$ test, $N=16$ subjects (no constraint) and $N=15$ (with constraint)). The average RTs in the control experiment $(438 \pm 18 \mathrm{~ms})$ were higher by about $100 \mathrm{~ms}$ compared to the main experiment $\left(341 \pm 11 \mathrm{~ms} ; P<10^{-4}, t\right.$ test). The success rates in the generalized trials of the control experiment were $67 \pm 4 \%\left(\mathrm{C}_{\mathrm{E}} \mathrm{C}_{\mathrm{H}}\right), 67 \pm 3 \%\left(\mathrm{C}_{\mathrm{E}} \mathrm{I}_{\mathrm{H}}\right), 80 \pm 2 \%$ $\left(\mathrm{I}_{\mathrm{E}} \mathrm{C}_{\mathrm{H}}\right)$ and $71 \pm 3 \%\left(\mathrm{I}_{\mathrm{E}} \mathrm{I}_{\mathrm{H}}\right)$. This means that the task performance for the generalized conditions dropped significantly compared to the main experiment $\left(P<10^{-5}, t\right.$ test, $N=16$ subjects (no constraint) and $N=15$ (with constraint)). Whereas in the main dataset there was no significant success rate difference between standard and generalized conditions ( $88 \pm 1$ vs. $86 \pm 1 \%, P>0.05$, paired $t$ test), the success rate in generalized conditions of the control experiment was significantly lower $(69 \pm 3 \%)$ than in the standard conditions $\left(80 \pm 2 \% ; P<10^{-5}\right.$, paired $t$ test). This means that performance difficulties in the control experiment mainly affected the generalized conditions.

The poor overall performance in the control experiment did not allow systematic comparisons between the results of the control and main experiments (data not shown). We attribute the idiosyncratic and non-conclusive results of the control experiment to the overall increased task difficulty, as indicated by a significant drop in performance and strong increase in average RTs (cf. "Discussion”).

\section{Discussion}

We tested how reach RTs, MTs and EVs are influenced by the spatial compatibility between a visual cue and the associated motor-goal in an eye- and/or hand-centered frame of reference. Our results show that there was not a global consistent influence of one single reference frame on multiple movement parameters, but different reference frames affected different behavioral parameters in a specific way. RTs were influenced by both, SRC in eye- and hand-reference frame, whereas MTs were influenced only by SRC in the hand-reference frame, and EVs were influenced only by $\mathrm{SRC}$ in the eye-reference frame.

\section{Spatial reference frames for reach planning}

Previous neurophysiology studies showed different predominant frames of reference for reach targets in different brain areas. Spatial tuning properties of most neurons in the parietal reach region (PRR; Batista et al. 1999; Buneo et al. 2002; Pesaran et al. 2006) as well as human imaging data from the posterior parietal cortex (Medendorp et al. 2003) fit best with a representation of the reach target in an eyereference frame. Neurons in parietal area 5 as well as a subpopulation of neurons in PRR showed hand-centered tuning (Buneo et al. 2002; Chang and Snyder 2010). In premotor areas, tuning properties of most neurons are driven by a combination of eye, hand and target position for reaching, whereas some are purely hand-centered, and others are purely eye-centered (Batista et al. 2007; Pesaran et al. 2006). How the coding of neurons in different reference frames translates into overt behavior is not obvious and might depend on the specific task.

Previous psychophysical experiments analysed systematic reach errors suggesting reach goal encoding in an eyecentered reference frame (Beurze et al. 2006; Henriques et al. 1998; Sorrento and Henriques 2008). Other experiments, which also analysed systematic reach errors, provided evidence for a hand-centered representation of reach goals (Bock and Eckmiller 1986; Gordon et al. 1994), or a combination of different reference frames (McGuire and Sabes 2009). This means, previous studies based on systematic reach errors showed idiosyncratic results. The diversity of findings could be due to the fact that reach errors can be expected to be influenced by different processes of motor planning and control or different sources of sensory input were differently weighted (McGuire and Sabes 2009).

Other studies have investigated the influence of reference frames on SR compatibility effects in reaction time 
(Lamberts et al. 1992; Nicoletti and Umilta 1989; Umilta and Liotti 1987). Lamberts et al. (1992) found joint compatibility effects of visual hemifield and relative position of two stimuli, in a task in which the response required buttonpresses of either the ipsi- or contralateral hand. In contrast to our study, this previous study could not compare the dissociated effects of an eye- and hand-reference frame, which was mostly investigated in neurophysiological studies. The reason for the inaccessibility of the hand-reference frame in the Lamberts study was the use of a dissociated workspace for visual cue and bimanual button-press responses (Lamberts et al. 1992), which is known to influence compatibility effects (Stins and Michaels 2000). Our results confirmed the influence of an eye-reference frame on RTs, and additionally show an influence of the hand-reference frame. Moreover, we could show distinct effects on other movement parameters, as discussed in the following paragraph.

\section{Kinematic reach parameters}

In contrast to RTs, MTs in our experiment were only influenced by compatibility in the hand-reference frame. The fact that MTs reflect parameters of movement planning and control might account for this, and is consistent with the notion, that hand-reference frames gain increasing importance the closer a brain structure is to the motor output (Batista et al. 2007; Pesaran et al. 2006).

EVs showed a significant effect of eye- but not of handreference frame. This effect was mainly induced by a reduced EV in trials with SR compatibility in both eye and hand-reference frame (Fig. 5a). Eye- and hand-compatible trials are characterized by the fact that cue and motor-goal were physically identical (same screen position). Despite the spatial cue being only briefly flashed (s. Methods), this might have led to a certain degree of visual guidance with reduced variability in movement trajectories, whereas in the other generalized task conditions the reach goal had to be spatially inferred, without the possibility of direct visual guidance.

\section{Spatial stimulus-response compatibility}

In the previous section, we interpreted and discussed our results in terms of eye- and hand-reference frames and their influence on SR compatibility effects. SR compatibility was defined as left/right compatibility of cue and motor-goal directions. However, there might be a more parsimonious explanation for the observed RT differences. RTs in the main experiment can be grouped to three different levels (Fig. 3). There was no difference between $\mathrm{C}_{\mathrm{E}} \mathrm{I}_{\mathrm{H}}$ reaches and $\mathrm{I}_{\mathrm{E}} \mathrm{C}_{\mathrm{H}}$ reaches $\left(P=0.9\right.$, paired $t$ test, $\left.\alpha_{\text {corr }}=0.0083\right)$. These three levels of RTs correlate with the distance between spatial cue and reach goal. A previous study (Stins and Michaels 2000) showed that the distance between cue and target can indeed influence RTs. Subjects are faster the closer the cue and target were together. In our task design, we cannot differentiate between the possibilities that RTs are explained by the combined compatibility in eye- and hand-reference frame, or by the distance between cue and reach goal.

Unlike RTs, MTs and EVs cannot be explained by the same dependency of absolute distance between cue and reach goal, since neither movement parameter scaled with this absolute distance. But the MT and EV results could possibly be explained by the compatibility of cue and goal eccentricity, i.e. the distance of the cue and the reach goal from the eye- or hand-fixation position (as depicted by the length of the arrows in Fig. 1b). According to this alternative view, compatibility of the direction of cue and reach goal would be irrelevant. Instead, reaches which are faster and more precise if cue and reach goal are compatible in eye eccentricity, i.e. if cue and goal are at the same distance from the eye-fixation, would indicate an influence of the eye-reference frame, Correspondingly, a compatibility effect of hand eccentricity would be taken as indication for an influence of the hand-reference frame. With this interpretation, RTs would have been determined by the compatibility of cue and goal eccentricity in an eye- and hand-reference frame. MTs would have been determined by the compatibility of cue and goal eccentricity in an eye-centered reference frame only. The latter means that reaches were faster if the reach goal position had the same distance from eye fixation as the cue compared to conditions in which the reach goal had a larger distance from eye fixation, despite identical hand eccentricity of the goal (=reach amplitude) in both conditions. EVs would have been determined by cue and reach goal eccentricity in a hand-centered reference frame. EVs were smaller if the cue had the same distance to the hand-fixation stimulus as the reach goal compared to conditions in which the cue had a larger distance from the hand-fixation stimulus, despite identical eye eccentricity of the cue in both conditions. We consider the possibility that MTs and EVs are explained by the compatibility of cue and goal eccentricities less plausible, since at least for the EVs it seems counter intuitive that they should be influenced by the distance of the cue from the hand-fixation stimulus independent of reach amplitude.

\section{Effect of eye movements}

In the control experiment, we wanted to test in how far involuntary saccades in the main experiment could have confounded our results. In standard trials of the control experiment, subjects performed similarly well as in the main experiment, and we found qualitatively the same results. However, results across subjects in the generalized conditions of the control experiment were rather idiosyncratic. We 
rule out that the RT results of the main experiment are explained by involuntary saccades aimed at the flashed spatial cue (or a following reorientation saccade toward the goal). If this was the case then we would have to expect that ocular fixation breaks mostly happened during or briefly after the presentation of the spatial cue, which was only the case for less than $19 \%$ of all ocular fixation breaks (3.7\% of all trials). Also, the standard pro-reaches and generalized anti-reaches denote trials with physically identical reaches, in which the cue was identical to the reach goal position and at the same distance from the fixation position. Yet, RTs in the main experiment were significantly different between these two conditions ( $P=0.0054$, paired $t$ test), again arguing against an effect induced by involuntary eye movements. Instead, we attribute the idiosyncratic results of the control experiment to the overall increased task difficulty, as indicated by significant drop in task performance and strongly increased overall reaction times (see "Results").

\section{Conclusions}

Different movement parameters, like reaction times, movement times, and endpoint variability, each reflect the stages of planning and control of a motor act to a different degree. We could show that eye- and hand-frames of reference have selective effects on the different movement parameters during goaldirected reaching. Hence, our results imply that eye- and hand-reference frames have distinct effects on the different stages of planning and control. In this sense, our results denote a psychophysical manifestation of the different observed reference frames at the different stages of neuronal processing, which putatively underlie different phases of overt motor behavior. During the planning stage, which we argue should be best reflected in the SRC effects on RTs, we found a twofold influence of eye- and hand-reference frame, without interaction, reminiscent of mixed reference frames of neurons in the parietal and frontal reach related sensorimotor areas.

Acknowledgments We thank Sadeem Jawhar and Constanze Lenschow for help in data collection, and Sina Plümer and Ludwig Ehrenreich for administrative and technical support. This work was supported by the Federal Ministry for Education and Research (BMBF, Germany) grants 01GQ0433 and 01GQ0814.

Open Access This article is distributed under the terms of the Creative Commons Attribution Noncommercial License which permits any noncommercial use, distribution, and reproduction in any medium, provided the original author(s) and source are credited.

\section{References}

Ariff G, Donchin O, Nanayakkara T, Shadmehr R (2002) A real-time state predictor in motor control: study of saccadic eye movements during unseen reaching movements. J Neurosci 22:7721-7729
Batista AP, Buneo CA, Snyder LH, Andersen RA (1999) Reach plans in eye-centered coordinates. Science 285:257-260

Batista AP, Santhanam G, Yu BM, Ryu SI, Afshar A, Shenoy KV (2007) Reference frames for reach planning in macaque dorsal premotor cortex. J Neurophysiol 98:966-983

Beurze SM, Van Pelt S, Medendorp WP (2006) Behavioral reference frames for planning human reaching movements. J Neurophysiol 96:352-362

Bock O, Eckmiller R (1986) Goal-directed arm movements in absence of visual guidance-evidence for amplitude rather than position control. Exp Brain Res 62:451-458

Buneo CA, Andersen RA (2006) The posterior parietal cortex: sensorimotor interface for the planning and online control of visually guided movements. Neuropsychologia 44:25942606

Buneo CA, Jarvis MR, Batista AP, Andersen RA (2002) Direct visuomotor transformations for reaching. Nature 416:632-636

Chang SWC, Snyder LH (2010) Idiosyncratic and systematic aspects of spatial representations in the macaque parietal cortex. PNAS 107:7951-7956

Crammond DJ, Kalaska JF (1994) Modulation of preparatory neuronal activity in dorsal premotor cortex due to stimulus-response compatibility. J Neurophysiol 71:1281-1284

de Jong R (1995) Strategical determinants of compatibility effects with task uncertainty. Acta Psychol 88:187-207

Duncan J (1977) Response selection errors in spatial choice reaction tasks. Q J Exp Psychol 29:415-423

Everling S, Dorris MC, Munoz DP (1998) Reflex suppression in the anti-saccade task is dependent on prestimulus neural processes. J Neurophysiol 80:1584-1589

Fischer B, Weber H (1992) Characteristics of antisaccades in man. Exp Brain Res 89:415-424

Fitts PM, Deininger RL (1954) S-R compatibility: correspondence among paired elements within stimulus and response codes. J Exp Psychol 48:483-492

Fitts PM, Seeger CM (1953) S R compatibility—spatial characteristics of stimulus and response codes. J Exp Psychol 46:199-210

Gail A, Andersen RA (2006) Neural dynamics in monkey parietal reach region reflect context-specific sensorimotor transformations. J Neurosci 26:9376-9384

Georgopoulos AP, Lurito JT, Petrides M, Schwartz AB, Massey JT (1989) Mental rotation of the neuronal population vector. Science 243:234-236

Gordon J, Ghilardi MF, Ghez C (1994) Accuracy of planar reaching movements. I. Independence of direction and extent variability. Exp Brain Res 99:97-111

Hallett PE (1978) Primary and secondary saccades to goals defined by instructions. Vision Res 18:1279-1296

Henriques DYP, Klier EM, Smith MA, Lowy D, Crawford JD (1998) Gaze-centered remapping of remembered visual space in an openloop pointing task. J Neurosci 18:1583-1594

Hommel B (1996) No prevalence of right-left over top-bottom spatial codes. Percept Psychophys 58:102-110

Khan AZ, Pisella L, Rossetti Y, Vighetto A, Crawford JD (2005) Impairment of gaze-centered updating of reach targets in bilateral parietal-occipital damaged patients. Cereb Cortex 15:15471560

Lamberts K, Tavernier G, d'Y dewalle G (1992) Effects of multiple reference points in spatial stimulus-response compatibility. Acta Psychol 79:115-130

McGuire LMM, Sabes PN (2009) Sensory transformations and the use of multiple reference frames for reach planning. Nat Neurosci 12:1056-1061

Medendorp WP, Goltz HC, Vilis T, Crawford JD (2003) Gaze-centered updating of visual space in human parietal cortex. J Neurosci 23:6209-6214 
Morin RE, Grant DA (1955) Learning and Performance on a key-pressing task as function of the degree of spatial stimulusresponse correspondence. J Exp Psychol 49:39-47

Nicoletti R, Umilta C (1984) Right-left prevalence in spatial compatibility. Percept Psychophys 35:333-343

Nicoletti R, Umilta C (1989) Splitting visual space with attention. J Exp Psychol Hum Percept Perform 15:164-169

Pesaran B, Nelson MJ, Andersen RA (2006) Dorsal premotor neurons encode the relative position of the hand, eye, and goal during reach planning. Neuron 51:125-134

Proctor RW, Vu KP (2002) Mixing location-irrelevant and locationrelevant trials: influence of stimulus mode on spatial compatibility effects. Mem Cognit 30:281-293
Shaffer LH (1965) Choice reaction with variable S-R mapping. J Exp Psychol 70:284-288

Sorrento GU, Henriques DYP (2008) Reference frame conversions for repeated arm movements. J Neurophysiol 99:2968-2984

Stins JF, Michaels CF (2000) Stimulus-response compatibility for absolute and relative spatial correspondence in reaching and in button pressing. Q J Exp Psychol Sect A Hum Exp Psychol 53:569-589

Umilta C, Liotti M (1987) Egocentric and relative spatial codes in S-R compatibility. Psychol Res Psychologische Forschung 49:81-90

Zhang M, Barash S (2000) Neuronal switching of sensorimotor transformations for antisaccades. Nature 408:971-975 